Relato de Caso

Case Report

Nair Kátia Nemr ${ }^{1}$

Marcia Simões-Zenari ${ }^{1}$

Tainá Soares Ferreira ${ }^{1}$

Heloisa Regina Fernandes ${ }^{1}$

Letícia Lessa Mansur ${ }^{1}$

Descritores

Miastenia gravis

Disfonia

Voz

Qualidade de vida

Diagnóstico

Keywords

Keywords Myasthenia gravis

Dysphonia

Voice

Quality of life

Diagnosis

Endereço para correspondência:

Marcia Simões-Zenari

Curso de Fonoaudiologia, Faculdade de Medicina, Universidade de São Paulo

R. Cipotânea, 51, Cidade Universitária, São Paulo (SP), Brasil, CEP: 05360-160.

E-mail: marciasz@usp.br

Recebido em: 21/12/2011

Aceito em: 19/02/2013

\section{Disfonia como principal queixa num quadro de miastenia grave: diagnóstico e fonoterapia}

\section{Dysphonia as the primary complaint in a case of myasthenia gravis: diagnosis and speech therapy}

Miastenia grave é uma doença autoimune que se manifesta por fraqueza e fadiga muscular progressivas. São frequentes os sintomas oculares e bulbares, dentre eles, a disfonia. Este artigo relata um caso de disfonia, cuja avaliação fonoaudiológica contribuiu com o diagnóstico de miastenia grave e seu tratamento. A paciente em questão procurou o atendimento fonoaudiológico com diagnóstico otorrinolaringológico de presbifonia. A avaliação perceptivo-auditiva e acústica da voz identificou alterações em respiração, fonte glótica e filtro/ressonância. Como alguns dados obtidos com a anamnese e observados nas provas vocais não se relacionavam diretamente com a presença de presbifonia, houve a necessidade de discussão do caso com o médico, quando ambos concluíram a necessidade de encaminhar a paciente para avaliação neurológica. O neurologista consultado levantou a hipótese diagnóstica de miastenia grave e solicitou exames. A paciente seguiu em acompanhamento fonoaudiológico e medicamentoso. Na reavaliação vocal, ocorrida cerca de dois meses após o início do tratamento, foi constatada melhora na qualidade vocal, com grande impacto na qualidade de vida. Este trabalho evidencia a importância da avaliação fonoaudiológica detalhada e da participação do fonoaudiólogo na equipe interdisciplinar.

\begin{abstract}
Myasthenia gravis is an autoimmune disease, manifested by progressive muscular weakness and fatigue. There are frequent ocular and bulbar symptoms, among them, dysphonia. This article reports a case of dysphonia that contributed to the diagnosis of myasthenia gravis through a speech evaluation. The patient sought speech therapy with the ENT diagnosis of presbyphonia. The perceptual voice assessment and acoustic analysis pointed out respiration, glottal voice source and resonance affections. Considering that some of the data obtained from anamnesis and vocal assessments were not directly related to presbyphonia, the speech therapist discussed the case with the physician and they both concluded it was necessary to refer the patient to a neurological evaluation. The neurologist then raised the diagnostic hypotheses of myasthenia gravis and requested further examinations. The patient underwent speech therapy and drug treatment. A vocal reassessment, which occurred two months after the initial treatment, showed improvement in voice quality, with great impact on quality of life. This article shows the importance of detailed clinical speech evaluation and participation of a speech therapist in an interdisciplinary team.
\end{abstract}

Trabalho realizado no Laboratório de Investigação Fonoaudiológica em Voz, Departamento de Fisioterapia, Fonoaudiologia e Terapia Ocupacional, Faculdade de Medicina, Universidade de São Paulo - USP São Paulo (SP), Brasil.

(1) Departamento de Fisioterapia, Fonoaudiologia e Terapia Ocupacional, Faculdade de Medicina, Universidade de São Paulo - USP - São Paulo (SP), Brasil.

Conflito de interesse: nada a declarar. 


\section{INTRODUÇÃO}

Miastenia grave é uma doença autoimune causada pela diminuição dos receptores de acetilcolina e perda dos receptores pós-sinápticos ${ }^{(1,2)}$. Manifesta-se por fraqueza e fadiga progressivas dos músculos esqueléticos ${ }^{(3)}$ e afeta entre 5 e 15 indivíduos a cada 100.000 , sendo $30 \%$ idosos $^{(2)}$. A prevalência é maior nas mulheres, numa proporção de cerca de $2: 1^{(4,5)}$. Há poucos dados clínicos e epidemiológicos sobre a ocorrência dessa doença no Brasil ${ }^{(5)}$.

A miastenia grave pode afetar um ou mais grupos musculares, sendo que o comprometimento do músculo elevador da pálpebra e do extraocular é bastante comum; então, sintomas como ptose palpebral e diplopia podem ser frequentes ${ }^{(3,6)}$. Sintomas bulbares como disfonia, disfagia, mastigação fraca, disartria e fraqueza na musculatura facial ${ }^{(1)}$ são também frequentes, especialmente quando a doença se manifesta após os 65 anos de idade ${ }^{(2)}$.

As dificuldades com a fala e com a deglutição aparecem como sintomas iniciais em mais de $27 \%$ dos $\operatorname{casos}^{(4)}$. Estudo com 40 indivíduos com miastenia grave encontrou disfonia como primeiro sintoma em $6 \%$ dos pacientes, sendo que, em $60 \%$ dos casos, aparece com a progressão da doença ${ }^{(3)}$. As alterações vocais encontradas usualmente nesses pacientes são: hipernasalidade, dificuldade em manter o pitch, fadiga vocal, afonia intermitente, estridor, rugosidade, incompetência glótica e mudanças na qualidade vocal ${ }^{(2,3,6)}$. Somadas à disfonia, imprecisão articulatória e alteração da fluência verbal também podem ser observadas ${ }^{(2)}$.

Em estudo brasileiro recente ${ }^{(5)}$, foram encontradas dificuldades na deglutição, e os distúrbios da voz foram mais comuns na faixa etária de 12 a 50 anos; acima dos 50 anos, a presença de disfonia foi tão frequente quanto a de diplopia.

O diagnóstico de miastenia grave é determinado a partir dos sinais clínicos e pode ser confirmado por eletromiografia em $95 \%$ dos $\operatorname{casos}^{(3)}$. E é fundamental que seja considerado o relato do paciente nesse diagnóstico. Com frequência, a disfonia não é associada com a doença durante o diagnóstico otorrinolaringológico ${ }^{(6)}$.

Se não for tratada, a miastenia grave pode incapacitar o indivíduo devido aos prejuízos que podem ocorrer nas terminações motoras ${ }^{(7)}$. O tratamento é usualmente medicamentoso ${ }^{(3)}$ ou, em alguns casos, cirúrgico, com melhora significativa dos sintomas $^{(6)}$ e até a remissão completa em alguns pacientes ${ }^{(7)}$. Há poucos dados disponíveis com relação à indicação de tratamentos associados, como fisioterapia e fonoterapia. Em revisão de literatura, a fisioterapia mostrou-se indispensável ${ }^{(7)}$, com melhora da performance motora, redução da fadiga e prevenção de complicações respiratórias generalizadas. A fonoterapia é indicada devido a mudanças na deglutição/mastigação, disfonia e dificuldades de fala, cuja melhora contribui para elevar a qualidade de vida dos pacientes ${ }^{(8,9)}$.

O objetivo deste artigo é relatar um caso de indivíduo idoso, no qual a avaliação fonoaudiológica foi fundamental para o encaminhamento da paciente para avaliação neurológica e, a partir daí, estabelecer o diagnóstico de miastenia grave e as condutas necessárias; além disso, pretende-se discutir os resultados do tratamento proposto.

\section{APRESENTAÇÃO DO CASO CLÍNICO}

Este estudo foi aprovado pelo Comitê de Ética da instituição (236/10). A paciente concordou com a realização e divulgação desta pesquisa e de seus resultados e assinou o termo de consentimento livre e esclarecido.

A paciente de 84 anos de idade foi encaminhada por médico otorrinolaringologista para avaliação fonoaudiológica por não ter sido possível fechar o diagnóstico apenas com o exame de laringoscopia.

Durante a anamnese fonoaudiológica realizada em nossa clínica-escola, a paciente mencionou que achava sua voz "horrível e desagradável" e que não conseguia falar alto, apresentando dificuldade em ser compreendida pelos outros. Os principais aspectos vocais indicados por ela foram: fadiga vocal, rouquidão, falhas na voz, tremor vocal e necessidade de fazer força para falar. Também se queixou de engasgos e tosse constantes. Relatou o uso diário de medicamentos prescritos pelo clínico que a acompanha devido a hipotireoidismo, episódios de refluxo gastroesofágico, diabetes, hipertensão arterial sistêmica e disfunção vestibular periférica.

Após a realização da anamnese, procedeu-se a avaliação vocal perceptivo-auditiva e acústica - análise qualitativa do espectro, avaliação da qualidade de vida em voz, da motricidade orofacial, da fala e das funções orais de mastigação e deglutição. Foram observadas alterações nos três sistemas de produção da voz: respiração, fonte glótica e filtro - tanto ressonância quanto articulação dos sons da fala. Durante as tarefas de avaliação, a paciente apresentou muita dificuldade em coordenar respiração e fala, seu pitch estava agravado, observou-se ataque vocal aspirado e loudness e frequência fundamental (f0) diminuídas. A extensão vocal mostrou-se reduzida, assim como os tempos máximos de fonação e a resistência vocal. Com aplicação do protocolo CAPE- $\mathrm{V}^{(10,11)}$, a disfonia foi considerada entre moderada e intensa, com astenia e rugosidade como os aspectos mais alterados, seguidos de instabilidade e soprosidade. A paciente apresentou engasgos e fadiga vocal com rápida deterioração da voz na fala espontânea, chegando à afonia. Foi observada importante imprecisão articulatória, principalmente em fonemas isolados, e dificuldade em coordenar os órgãos fonoarticulatórios e em sustentar sons fricativos. A frequência fundamental média foi $107,52 \mathrm{~Hz}$ (Tabela 1).

A análise espectrográfica acústica foi realizada com uso do programa Spectrogram e a extração da frequência fundamental, com o programa Praat. O traçado mostrou harmônicos parcialmente visíveis, presença de ruído entre os harmônicos, substituição de harmônicos por ruído, interrupções, bifurcações, irregularidade gráfica e série de harmônicos reduzida (Figura 1).

Observou-se que as alterações vocais produziam impacto negativo importante na qualidade de vida da paciente, limitando sua participação em eventos sociais e familiares. No Questionário de Qualidade de Vida em Voz $(\mathrm{QVV})^{(12)}$, sua pontuação foi 25 numa escala de 0 a 100, em que o valor máximo representa melhor qualidade de vida relacionada à voz.

Após a avaliação fonoaudiológica, a paciente foi encaminhada para avaliação laringológica no ambulatório de otorrinolaringologia do hospital de referência do serviço, o que faz parte dos procedimentos de 
rotina; o diagnóstico foi de presbifonia. Entretanto, como a avaliação fonoaudiológica havia levantado aspectos que não eram compatíveis apenas com esse diagnóstico como importante fadiga vocal, disartria e disfagia, a equipe discutiu o caso e decidiu encaminhar a paciente para avaliação neurológica no mesmo hospital. O neurologista que a avaliou considerou os sinais clínicos suficientemente sugestivos para a hipótese diagnóstica de miastenia grave, por isso, iniciou o tratamento medicamentoso em paralelo à realização dos exames. Posteriormente, esse diagnóstico foi confirmado.

A paciente seguiu programa de fonoterapia semanal com ênfase em técnicas para fechamento glótico, articulação dos sons da fala e melhora da resistência e intensidade vocal. Para evitar que a paciente entrasse em fadiga vocal, os exercícios selecionados foram distribuídos em cada sessão de maneira a intercalar técnicas de fonte glótica com outras que não envolvessem a produção vocal, como as respiratórias e articulatórias com sons surdos. Ao menor sinal de perda da qualidade vocal/fadiga vocal, os exercícios eram interrompidos. Os exercícios de fonte glótica não foram indicados para a prática domiciliar no início do tratamento. Como a fadiga vocal foi um dos sintomas que melhorou rapidamente devido ao uso do medicamento, aos poucos esse controle passou a ser menos necessário.

Após dois meses de fonoterapia e de uso do medicamento, uma nova avaliação clínica da voz e da fala foi realizada, quando ficou evidente a melhora nas alterações inicialmente identificadas. A rugosidade passou para grau moderado, e astenia e instabilidade passaram para grau leve a moderado. Apenas a presença de soprosidade ficou mais evidente, o que é compatível com a maior intensidade vocal apresentada pela paciente na presença de coaptação glótica incompleta. Além disso, o tempo máximo de fonação e a resistência vocal melhoraram, e a fadiga vocal diminuiu; também a frequência fundamental mostrou-se mais elevada (Tabela 1).

No traçado espectrográfico, as bifurcações e interrupções observadas inicialmente não estavam mais presentes, além de se observarem melhor definição dos harmônicos e maior regularidade. Apenas a presença de ruído entre os harmônicos e substituição de harmônicos por ruído nas frequências acima de $2.000 \mathrm{~Hz}$ aumentou, o que é compatível com a maior presença de soprosidade relatada anteriormente (Figura 2).

Tabela 1. Aspectos da avaliação fonoaudiológica pré e pós-fonoterapia

\begin{tabular}{lcc}
\hline Aspectos avaliados & \multicolumn{2}{c}{ Momento da avaliação } \\
\hline Qualidade vocal, protocolo CAPE-V & Pré-fonoterapia & Pós-fonoterapia \\
Coordenação respiração-fala & predominam astenia e rugosidade & Disfonia de leve a moderada; \\
Tempos máximos de fonação & Alterada & predominam soprosidade e rugosidade \\
Frequência fundamental média & Reduzidos & Adequada \\
Resistência vocal & $107,52 \mathrm{~Hz}$ & $152,74 \mathrm{~Hz}$ \\
Extensão vocal & Diminuída, com episódios de afonia & Adequada \\
Ataque vocal & Reduzida & Adequada \\
Pitch & Aspirado & Adequado \\
Loudness & Agravado & Adequado \\
& Diminuída & Adequada \\
Espectrografia & Principalmente bifurcações, substituição de & Principalmente série de harmônicos \\
& harmônico por ruído em frequências baixas; & reduzida; presença de ruído entre harmônicos \\
Articulação dos sons da fala & irregularidade e série de harmônicos reduzida & e acima de 2.000 Hz devido à maior presença \\
Disfagia & Imprecisa & de ar não sonorizado \\
Índice QVV & Engasgos frequentes & Mais precisa \\
\hline
\end{tabular}

Legenda: $\mathrm{QVV}=$ Qualidade de Vida em Voz

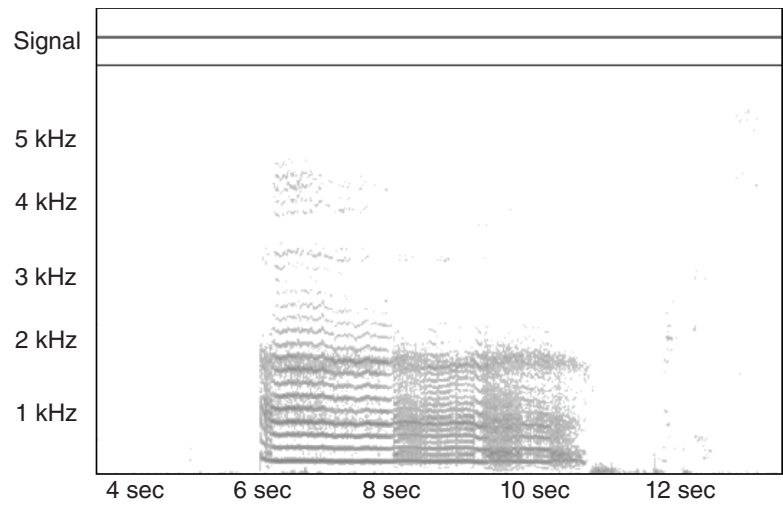

Figura 1. Imagem ilustrativa do espectrograma referente à emissão da vogal /a/ no momento pré-tratamento, programa Spectrogram

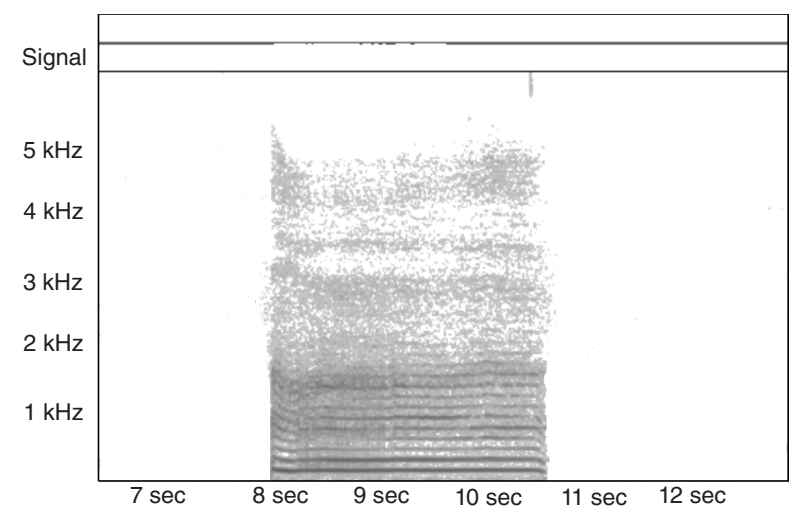

Figura 2. Imagem ilustrativa do espectrograma referente à emissão da vogal /a/ no momento pós-tratamento, programa Spectrogram 
Contudo, a melhora mais significativa se deu em relação à qualidade de vida relacionada à voz, expressa por meio dos relatos da paciente e também do índice QVV, que passou de 25 para 60.

É importante indicar que, durante a fonoterapia, foi realizada avaliação auditiva, e a paciente recebeu o diagnóstico de presbiacusia. Esse fato pode explicar sua dificuldade em perceber alguns aspectos da produção vocal.

\section{DISCUSSÃO}

A apresentação deste caso evidencia o quanto a avaliação fonoaudiológica foi importante para que a equipe pudesse chegar ao diagnóstico correto e iniciar rapidamente o tratamento, o que não seria possível apenas com os diagnósticos otorrinolaringológicos isolados.

Aspectos vocais decorrentes da miastenia grave estão relacionados à fadiga e fraqueza dos músculos adutores e tensor das pregas vocais. Disfagia pode estar associada às afecções dos músculos da faringe e esôfago, e disartria e imprecisão articulatória podem ser justificadas pela fraqueza dos músculos da língua e palato ${ }^{(2,3)}$. Valorizar tanto os aspectos clínicos como o relato da paciente foi crucial para definir o tratamento adequado para este caso. A fonoterapia associada ao tratamento medicamentoso possibilitou que muitos aspectos alterados melhorassem rapidamente.

Houve importante melhora na qualidade de vida da paciente, o que foi muito significativo para ela, pois possibilitou ganhos na inteligibilidade e motivação para interações sociais.

Os dados encontrados na análise inicial do traçado espectrográfico estão de acordo com a literatura sobre disartria: instabilidade no traçado espectrográfico com ausência de harmônicos nas frequências altas devido à fraqueza e lentidão ou falta de coordenação dos músculos laríngeos, além de distribuição irregular de energia no trato vocal ${ }^{(13)}$.

O diagnóstico precoce e o início ágil do tratamento multiprofissional possibilitam melhora dos sinais e sintomas da miastenia grave e, em muitos casos, até remissão total destes. Para os pacientes que apresentam disfagia associada a esta doença, esses fatores são essenciais para prevenir aspiração, desidratação e desnutriçãa ${ }^{(14)}$.

Neste caso, o tratamento medicamentoso levou à rápida diminuição dos sintomas vocais e da fadiga durante a fala e proporcionou plena participação da paciente na fonoterapia. Além disso, a fadiga vocal foi considerada no planejamento terapêutico, principalmente no que se refere à distribuição dos exercícios durante a sessão e atenção do terapeuta para o menor sinal de sua presença.

Os resultados superaram as expectativas da paciente e dos profissionais envolvidos, principalmente pelo fato de haver outras doenças associadas com impacto na voz, como refluxo gastroesofágico, presbiacusia e hipotireoidismo, esta última muito comum em casos de miastenia grave ${ }^{(15)}$. Atenção especial aos aspectos descritos deve ser dada pelos profissionais na análise para diagnóstico diferencial.

\section{COMENTÁRIOS FINAIS}

Este estudo de caso reitera a importância da avaliação fonoaudiológica para o diagnóstico de pacientes com alterações de voz e fala. A combinação entre laringoscopia, sinais clínicos, aspectos relatados pelos pacientes e o diálogo interdisciplinar permitiu a compreensão completa do quadro e auxiliou na definição do diagnóstico com o encaminhamento necessário e a determinação dos cuidados adequados a esta paciente. Os resultados obtidos tanto em relação ao tratamento médico quanto fonoterápico foram altamente positivos e reforçam a relevância deste último não apenas para prevenir complicações, mas para proporcionar melhor qualidade de vida.

\footnotetext{
* NKN e LLM foram responsáveis pelo projeto e delineamento do estudo e orientação geral das etapas de execução e elaboração do manuscrito; MSZ supervisionou a coleta de dados e colaborou com a análise dos dados e com a elaboração do manuscrito; TSF e HRF responsáveis pela coleta e tabulação dos dados.
}

\section{REFERÊNCIAS}

1. Kanemaru SI, Fukushima H, Kojima H, Kaneko KI, Yamashita M, Ito J. A case report of myasthenia gravis localized to the larynx. Auris Nasus Larynx. 2007;34(3):401-3.

2. Montero-Odasso M. Dysphonia as first symptom of late-onset myasthenia gravis. J General Internal Medicine. 2006;21(6):C4-C6.

3. Mao VH, Abaza, M, Spiegel JR, Mandel S, Hawshaw M, Heuer RJ, et al. Laryngeal myasthenia gravis: report of 40 cases. J Voice. 2001;15(1):122-30.

4. Jacobson DL, Gange SJ, Rose NR, Graham NM. Epidemiology and estimated population burden of selected autoimmune diseases in the United States. Clin Immunol Immunopathol. 1997;84(3):223-43.

5. Aguiar AAX, Carvalho AF, Costa CMC, Fernandes JMA, D'Almeida JAC, Furtado LETA, et al. Myasthenia gravis in Ceará, Brazil. Arq Neuropsiquiatr. 2010;68(6):843-8.

6. Liu WB, Xia Q, Men LN, Wu ZK, Huang RX. Dysphonia as a primary manifestation in myasthenia gravis (MG): a retrospective review of 7 cases among 1520 MG patients. J Neurol Sci. 2007;260(1-2):16-22.

7. Santos FRM, Tiago APPP, Fonseca AL, Christofoletti G. Revisão da fisioterapia na miastenia grave. Revista Movimenta 2008;1(1):16-22.

8. Penatti CT, Silva IA, Domenis DR, Okubo PCMI. Atuação fonoaudiológica na miastenia grave auto-imune adquirida: descrição de um caso. Rev Soc Bras Fonoaudiol. 2008;(Suppl):217.

9. Bassi AKZ, Melo TT, Pedraça LS, Cortêz ASS, Silva VB, Rodrigues LB, et al. Características fonoaudiológicas e audiológicas de uma paciente com miastenia grave: relato de caso. Rev Soc Bras Fonoaudiol. 2009;(Suppl):1929.

10. Kempster GB, Gerratt BR, Verdolini Abbott K, Barkmeier-Kraemer J, Hillman RE. Consensus Auditory-Perceptual Evaluation of Voice: development of a standardized clinical protocol. Am J Speech Lang Pathol. 2009;18(2):124-32.

11. Behlau M. Consensus Auditory - Perceptual Evaluation of Voice (CAPE-V). ASHA 2003 [Refletindo sobre o novo]. Rev Soc Bras Fonoaudiol. 2004;9(3):187-9.

12. Gasparini G, Behlau M. Quality of Life: Validation of the Brazilian Version of the Voice-Related Quality of Life (V-RQOL) Measure. J Voice. 2009;23(1):76-81.

13. Ortiz KZ, Carrillo L. Comparação entre as análises auditiva e acústica nas disartrias. Rev Soc Bras Fonoaudiol. 2008;13(4):325-31.

14. Oda AL, Chiappetta ALML, Annes M, Marchesan IQ, Oliveira ASB. Avaliação clínica, endoscópica e manométrica da deglutição em pacientes com miastenia grave autoimune adquirida. Arq Neuropsiquiatr. 2002;60(4):986-95.

15. Bichuetti DB, Barros TM, Oliveira EML, Annes M, Gabbai AA. Demyelinating disease in patients with myasthenia gravis. Arq Neuropsiquiatr. 2008;66(1):5-7. 